\title{
A study on torsional stiffness of the competition go-kart frame
}

\author{
C.-C. Liang, C.-H. Yu \& C.-C. Wu \\ Department of Mechanical and Automation Engineering, \\ Da-Yeh University, Chang-Hua, Taiwan, Republic of China
}

\begin{abstract}
The construction of the competition go-kart must satisfy the philosophy of extreme simplicity: no redundant part is added and the go-kart frame is similar to the chassis of a sedan. Accordingly, the design of each part on the kart has to contribute to its performance. Due to the absence of a differential gear and suspension system, the frame must be responsible for offering proper rigidity and torsional stiffness to the kart and producing load transfers during cornering. Therefore, how to build a good kart frame is very important to go-kart design. In this paper, the torsional stiffness of the go-kart frame is analyzed in detail by using non-linear, explicit dynamic code LS-DYNA 3D. The kart frame presented by Solazzi is adopted to be the parent model, and two design concepts are investigated to improve the torsional stiffness of the kart frame. The first one is the arrangement of extra members at suitable positions on the frame, and nine different types of frame are studied. The second one is to increase the width between two kingpins, and four different types are studied. The torsional stiffness of each frame type is determined and compared with each other. The result shows that the torsional stiffness of the frames with larger kingpin width is better than those with extra members. Specifically, the one with width of 828 $\mathrm{mm}$ is the best type of frame, and the torsional stiffness is $31.03 \%$ higher than the original. In addition, among the frames with extra members, the one with a right-inclined member arranged in front of the centre member is the best design, and the torsional stiffness is $20.18 \%$ higher than the original. It is believed that the design concepts presented in this research will provide a useful reference for the design and manufacturing of an optimum go-kart frame.
\end{abstract}

Keywords: go-kart, torsional stiffness, LS-DYNA $3 D$. 


\section{Introduction}

Most of the fans of vehicle racing understand that the go-kart (also called karting) is the simplest formula racing in the world, and most of the F1 drivers began their careers by taking part in karting competitions. In addition, the gokart is also a popular recreation in the world, because it is suitable for people of all ages and can be divided into several kinds according to different purposes, such as recreation and competition. In general, the most popular go-kart has no differential gear and suspension system, as shown in Figure 1, so that its dynamic behavior is different from those of standard vehicles. For this reason, the inner wheels of the kart would rise during cornering. Therefore, the frame of a kart must be able to offer proper bending rigidity and torsional stiffness to improve the handling stability and to absorb the serious vibration produced during driving. Since research work on racing engineering is an absolute secret and tunings of go-kart are mainly experience based, the literature concerning the research on go-karting is very limited. The study on how to determine a frame with adequate torsional stiffness is hence of importance to the go-kart design.

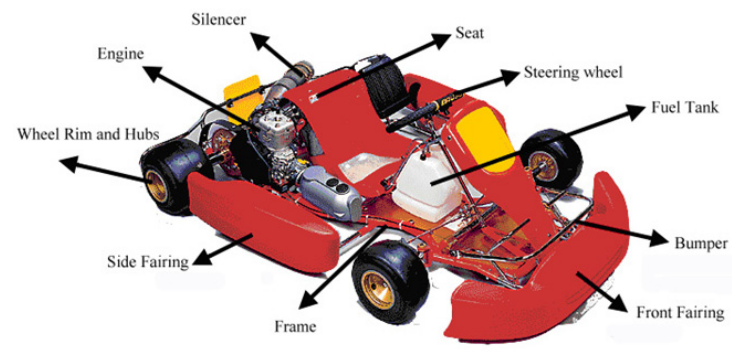

Figure 1: The construction of a competition go-kart.

In this research, a detail analysis on how to improve torsional stiffness of the go-kart frame by using commercial code LS-DYNA 3D is presented. The Solazzi's kart frame model is used as the original model to study two design concepts of improving torsional stiffness: arrangement of extra members at suitable positions and increasing kingpin width of the frame. It is believed that the concepts presented in this research will provide a good reference on the tuning of the go-kart frame to racing technicians.

\section{Configuration of the go-kart frame}

The general configuration of the go-kart frame is shown in Figure 2. The CIKFIA technical regulations [1] published the general design concepts of competition go-kart in every respect, and clearly classified competition go-kart into several classes according to the displacements and some special regulations. The technical specifications of a kart are shown in Table 1. The frame is the main supporting part of the whole kart. It must be stiff sufficiently to absorb the 
charges produced when the kart is in motion. The frame must be magnetized steel tubular construction with a cylindrical section; it should be in an integral piece with welded parts not to be dismounted. In order to keep the flexibility of the frame, the stress caused by the dynamic loads should not exceed the elastic limits.

Tubes that are welded together to form the structure of the frame are made of magnetic structural steel or structural steel alloy. The frame can be tuned to adapt to the track by adjusting the middle, rear, and longitudinal part to achieve the suitable stiffness. Extra members on both sides of the seat can change the torsional stiffness of the frame. Since the karts will suffer from fatigue at high speed cornering, the working life of a frame is about 1 to 2 years. However, drivers of competition go-kart would rather use a new frame every 2 or 3 races to assure the handling performance being in the optimal conditions.

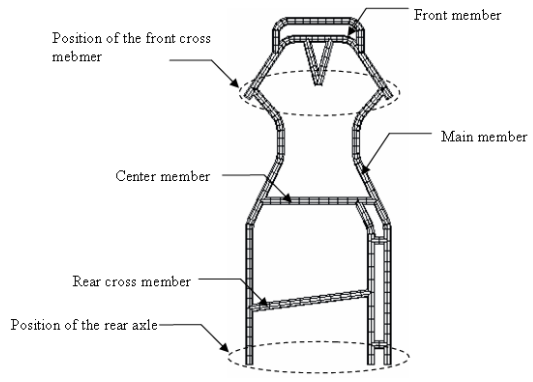

Figure 2: Illustration of each member of the go-kart frame.

Table 1: $\quad$ Technical specifications of the go-kart frame [1].

\begin{tabular}{|l|l|l|}
\hline \multicolumn{2}{|c|}{ Items } & \multicolumn{1}{c|}{ Specification } \\
\hline A & Wheelbase & $\begin{array}{l}\text { Min.: } 101 \mathrm{~cm} \text { (except in Superkart: } 106 \mathrm{~cm}) \\
\text { Max.: } 127 \mathrm{~cm}\end{array}$ \\
\hline B & Track & At least $2 / 3$ of the wheelbase used \\
C & Overall length & $\begin{array}{l}182 \mathrm{~cm} \text { max. without a front and } / \text { or rear fairing } \\
\text { (except for long circuits: } 210 \mathrm{~cm} \text { max.) }\end{array}$ \\
\hline D & Overall width & $\begin{array}{l}140 \mathrm{~cm} \text { maximum, except in ICA-J: } 136 \mathrm{~cm} \\
\text { max. }\end{array}$ \\
\hline
\end{tabular}

\section{Finite element model in the simulation of twisting test}

In order to accurately simulate the whole twisting test and the improving design study of go-kart frame, the finite element analysis software, the non-linear, explicit code LS-DYNA 3D is adopted to validate the precision of the frame 
model built in this paper. LS-DYNA 3D is a commercial code including preprocessing FEMB (Finite Element Method Builder), solver, and post-processing LS-POST. It is based on the finite element method, and is a three-dimensional analysis code, in which all element types and material models are available. Lagrangian processor and implicit method are used in this paper. In FEMB, the elements, mesh techniques, materials, and boundary conditions of the frame model are defined.

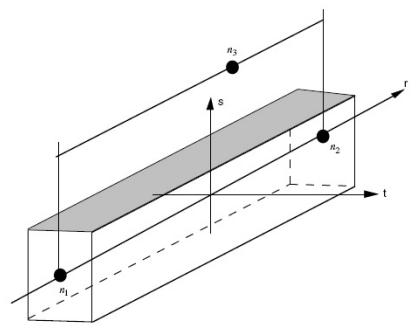

Figure 3: Lagrangian beam element.

In this paper, the beam element, as shown in Figure 3, is selected to build the frame model. Each beam element consists of three nodes, and each node has 6 DOFs (degrees of freedom), including 3 translational and 3 rotational DOFs. The frame model is then divided with line mesh, and the sizes of the elements depend on the length of the lines. Several material models are available in LSDYNA 3D to simulate a wide range of linear and non-linear material behaviors. In which, elastoplastic material is assigned to the frame model in this paper. The boundary conditions should be set to simulate the real analysis of torsional stiffness. The translational and rotational DOFs at the two points on both sides of the rear end frame are constrained (i.e., $U_{x}=U_{y}=U_{z}=0, \theta_{x}=\theta_{y}=\theta_{z}=0$, at points $\mathrm{D}$ and $\mathrm{E}$, as shown in Figure 4). The horizontal translational and three rotational DOFs at the center point (point $\mathrm{C}$ in Figure 4) of the front part of the frame are also constrained (i.e., $U_{x}=U_{y}=0, \theta_{x}=\theta_{y}=\theta_{z}=0$ ). In the simulation of twisting test, the vertical displacement $(\Delta y)$ of the two kingpins is determined by using LS-DYNA 3D.

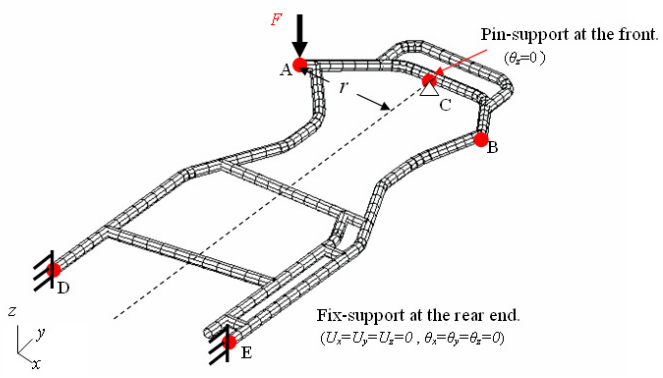

Figure 4: Determining the torsional stiffness of the go-kart frame by using the twisting test. 


\section{A conceptual design study of improving torsional stiffness on the go-kart frame}

The dimensions of the kart frame must comply with the regulations of CIK-FIA, but even slight modification on the structure of the frame can obviously influence the handling performance and the torsional stiffness of a kart.

Table 2: $\quad$ Different types of frame with extra members mounted.

\begin{tabular}{|c|c|c|c|c|}
\hline Model (a) & Model (b) & Model (c) & Model (d) & Model (e) \\
\hline & & & & \\
\hline Model (f) & Model (g) & Model (h) & Model (i) & Model (j) \\
\hline & & & \\
\hline
\end{tabular}

Note: (- - ) The dashed circles show the position of the extra members on the frame.

Table 3: $\quad$ Different types of frame with extra members mounted.

\begin{tabular}{|c|c|c|c|c|}
\hline Model (a) & Model (k) & Model (1) & Model (m) & Model (n) \\
\hline & & &
\end{tabular}

In this paper, the frame model built by Solazzi [2] is taken as the parent model and then several types of frame are built and compared to yield the optimum design. Arrangement of extra members at suitable positions on the frame as shown in Table 2, and increasing the kingpin width of the frame as shown in Table 3 are two design concepts studied in this paper. Since the go-kart frame is an asymmetric structure, the torsional stiffness of the frame will be different when the kart is cornering right or left, by implementing the twisting 
test, the vertical force $\left(F_{z f o}\right)$ is applied on the left and the right kingpin, respectively. Furthermore, the vertical force applied on the left kingpin is to simulate the kart cornering right, and kart cornering left is by the vertical force applied on the right kingpin.

\subsection{Different frames with extra members arranged}

Table 2 shows ten different types of frame, Model (a) is the original model built by Solazzi [2], and others are with extra members arranged (dashed circles). The remaining models can be divided into three groups: the first one includes models (b) and (c), which are arranged with longitudinal members on the frame; the second one includes models (d) and (e), which are arranged with horizontal members; the last one includes models (f), (g), (h), (i), and (j), which are arranged with inclined members. Tables 4 and 5 show the torsional stiffness of the models described as follows:

(1) Model (a) is the original model built by Solazzi [2] with weight of $10.21 \mathrm{~kg}$, and is the basis of modifications of other models.

(2) Model (b) is arranged with two longitudinal members near the front axle. It has the weight of $11.06 \mathrm{~kg}$. The torsional stiffness is $15.3 \%$ and $15.5 \%$ higher than the original while the force is applied on the left and right kingpins, respectively.

Table 4: The comparison of torsional stiffness for different types of frame with vertical force applied on left kingpin.

\begin{tabular}{|c|c|r|r|r|}
\hline & Vertical & Angle of twist & Torsional stiffness & $\begin{array}{c}\text { Percentage of } \\
\text { increment } \\
(\%)\end{array}$ \\
\hline Model & displacement & $(\Delta \theta)$ & $\left(K_{T}\right) N \cdot m m /$ deg & 0 \\
\hline Model (a) & 6.8428 & 0.623513 & 193619.7 & 15.2111 \\
\hline Model (b) & 5.9393 & 0.541192 & 223071.4 & 8.782695 \\
\hline Model (c) & 6.2903 & 0.573173 & 210624.7 & 11.41455 \\
\hline Model (d) & 6.1417 & 0.559633 & 215720.5 & 19.77871 \\
\hline Model (e) & 5.7128 & 0.520554 & 231915.2 & 6.374746 \\
\hline Model (f) & 6.4327 & 0.586148 & 205962.5 & 20.09191 \\
\hline Model (g) & 5.6979 & 0.519196 & 232521.6 & 14.82447 \\
\hline Model (h) & 5.9593 & 0.543014 & 222322.8 & 19.23815 \\
\hline Model (i) & 5.7387 & 0.522914 & 230868.5 & 13.2679 \\
\hline Model (j) & 6.0412 & 0.550476 & 219309 & 7.146158 \\
\hline \hline Model (k) & 7.4425 & 0.628202 & 207456.1 & 14.70625 \\
\hline Model (l) & 8.0139 & 0.630024 & 222093.9 & 22.6136 \\
\hline Model(m) & 8.5611 & 0.629831 & 237404.1 & 30.85281 \\
\hline Model (n) & 9.0852 & 0.628064 & & \\
\hline
\end{tabular}

Note: Percentage of increment $(\%)=\left(\left[K_{T}\right.\right.$ of model $\left.\mathrm{x}\right]-\left[K_{T}\right.$ of model a $\left.]\right) / K_{T}$ of model a $(\mathrm{x}=\mathrm{a} \sim \mathrm{n})$ 
Table 5: Comparison of torsional stiffness for different types of frame with vertical force applied on the right kingpin.

\begin{tabular}{|c|c|c|c|c|}
\hline & Vertical & Angle of twist & Torsional stiffness & $\begin{array}{c}\text { Percentage of } \\
\text { increment } \\
(\%)\end{array}$ \\
\hline Model & displacement & $(\Delta \theta)$ & $\left(K_{T}\right) N \cdot m m / d e g$ & 0 \\
\hline Model (a) & 6.9694 & 0.635048 & 190102.8 & 15.53738 \\
\hline Model (b) & 6.0321 & 0.549647 & 219639.8 & 8.865587 \\
\hline Model (c) & 6.4018 & 0.583332 & 206956.5 & 11.63277 \\
\hline Model (d) & 6.2431 & 0.568872 & 212217.0 & 19.90219 \\
\hline Model (e) & 5.8125 & 0.529638 & 227937.4 & 7.58012 \\
\hline Model (f) & 6.4783 & 0.590302 & 204512.8 & 15.48186 \\
\hline Model (g) & 6.035 & 0.549911 & 219534.3 & 19.8692 \\
\hline Model (h) & 5.8141 & 0.529784 & 227874.7 & 14.03455 \\
\hline Model (i) & 6.1116 & 0.556891 & 216782.9 & 18.87557 \\
\hline Model (j) & 5.8627 & 0.534212 & 225985.8 & 7.109388 \\
\hline \hline Model (k) & 7.5828 & 0.640044 & 203617.9 & 14.60754 \\
\hline Model (1) & 8.1692 & 0.642232 & 217872.1 & 22.35113 \\
\hline Model(m) & 8.7382 & 0.642859 & 232592.9 & 30.45232 \\
\hline Model (n) & 9.2817 & 0.641647 & 247993.5 & \\
\hline
\end{tabular}

Note: Percentage of increment $(\%)=\left(\left[K_{T}\right.\right.$ of model $\left.\mathrm{x}\right]-\left[K_{T}\right.$ of model $\left.\left.\mathrm{a}\right]\right) / K_{T}$ of model a $(\mathrm{x}=\mathrm{a} \sim \mathrm{n})$

(3) Model (c) is arranged with two longitudinal members in front of the center member. It has the weight of $10.73 \mathrm{~kg}$. The torsional stiffness is $8.9 \%$ higher than the original while the force is applied on the left kingpin, but is the same while the force is applied on the right kingpin.

(4) Model (d) is arranged with a horizontal member in front of the main members. It has the weight of $10.87 \mathrm{~kg}$. The torsional stiffness is $11.45 \%$ and $11.6 \%$ higher than the original while the force is applied on the left and right kingpins, respectively. Actually, the results are nearly the same.

(5) Model (e) is arranged with a horizontal member at the main members. It has the weight of $10.74 \mathrm{~kg}$. The torsional stiffness is $19.86 \%$ and $19.9 \%$ higher than the original while the force is applied on the left and right kingpins, respectively. Actually, the results are nearly the same.

(6) Model (f) is arranged with an inclined member in front of the rear cross member. It has the weight of $10.91 \mathrm{~kg}$. The torsional stiffness is $6.4 \%$ and $7.6 \%$ higher than the original while the force is applied on the left and right kingpins, respectively.

(7) Model (g) is arranged with a right-inclined member in front of the center member. It has the weight of $11.01 \mathrm{~kg}$. The torsional stiffness is $20.18 \%$ and $15.5 \%$ higher than the original while the force is applied on the left and right kingpins, respectively. 
(8) Model (h) is arranged with a left-inclined member in front of the center member. It has the weight of $11.01 \mathrm{~kg}$. The torsional stiffness is $14.89 \%$ and $19.9 \%$ higher than the original while the force is applied on the left and right kingpins, respectively.

(9) Model (i) is arranged with a right-inclined member at the main members. It has the weight of $10.95 \mathrm{~kg}$. The torsional stiffness is $19.33 \%$ and $14.03 \%$ higher than the original while the force is applied on the left and right kingpins, respectively.

(10) Model (j) is arranged with a left-inclined member at the main members. It has the weight of $10.95 \mathrm{~kg}$. The torsional stiffness is $13.33 \%$ and $18.88 \%$ higher than the original while the force is applied on the left and right kingpins, respectively.

\subsection{Different frames with width increased}

Table 3 shows the five different frame types; four of them are with increased kingpin width. Model (a) is the original model built by Solazzi, and Tables 4 and 5 show the calculated torsional stiffness of the models.

The increment of width from model $(\mathrm{k})$ to model $(\mathrm{n})$ is $50 \mathrm{~mm}$, so the widths are $678,728,778$, and $828 \mathrm{~mm}$, and the weights are $10.55,10.90,11.25$, and $11.61 \mathrm{~kg}$, for the four models, respectively. The torsional stiffness are $7.20 \%$, $14.82 \%, 22.76 \%$, and $31.03 \%$ and are $7.11 \%, 14.61 \%, 22.35 \%$, and $30.45 \%$ higher than the original model while the force is applied on the left and right kingpin, respectively. One can realize the increasing percentage of the torsional stiffness is about 7\% for each 50-mm increment of the kingpin width.

\subsection{Comparison of torsional stiffness of twisting test}

Tables 4 and 5 show the results of the twisting test. It is noted that different positions of the extra members and different widths affect the torsional stiffness and the weight of the kart frame. Some important facts are drawn as follows:

The torsional stiffness for the nine different types of frame structures with extra members are calculated to obtain the best one:

1. Models (b) and (c) are arranged with longitudinal members, and the torsional stiffness of model (b) is higher than that of model (c) no matter which kingpin the force is applied. One sees that longitudinal members arranged like model (b) can have better result, and it will nearly be the same when the kart is cornering right or left.

2. Models (d) and (e) are arranged with horizontal members, and the torsional stiffness of model (e) is higher than that of model (d) no matter which kingpin the force is applied. One can see that arranging horizontal members at the middle part of the main frame structure result in higher stiffness than those arranging in the front part. Extra horizontal members can also improve handling performance both in cornering right or left.

3. Models (f), (g), (h), (i), and (j) are arranged with inclined members, and Model ( $\mathrm{g}$ ) results in highest torsional stiffness while the force is applied on the left kingpin. On the other hand, model (h) will be the one achieving the 
highest stiffness while the force is applied on the right kingpin. Both model (g) and (h) can only improve handling performance in an alternative way of the kart cornering right or left.

4. Among the nine types of frame, model (e) and (g) will achieve the highest torsional stiffness while the force is applied on the right and left kingpin, respectively. However, model (e) will compromise to have better improvement on handling performance in both kart cornering right and left at the same time.

5. It should be noted that the torsional stiffness of the frames with longitudinal members or horizontal members added to the frame are independent of the force applying on which kingpin.

6. The torsional stiffness of the frames added with right/left inclined members depends upon which kingpin force is applied. One can know that frames arranged with right inclined-members behave better than those arranged with left-inclined members while the force is applied on the left kingpin.

Similarly, the torsional stiffness for each of four different types of frame structures with horizontal width increase is also calculated to obtain the best one. Among these four frame types, model (n) has the highest torsional stiffness no matter which kingpin the force is applied on. Furthermore, one may realize the torsional stiffness of the frame is proportional to the horizontal width of the frame. The torsional stiffness of the frames with width increased is also independent of which side the force is applied. However, the maximum width of the frame is regulated as $1400 \mathrm{~mm}$.

Biancolini et al. [3] recommended that the torsional stiffness for go-kart frame should be at least $165000 \sim 169000(\mathrm{~N} . \mathrm{mm} / \mathrm{deg})$. From both the fourth column of Tables 4 and 5, one can see that all the models suggested in this research have exceeded the recommended minimum torsional stiffness.

\section{Conclusions}

The torsional stiffness of a go-kart frame must be able to compensate the fact of no differential gear by producing load transfers during cornering. It is one of the important factors influencing the handling performance of a kart. According to the analysis presented in this research, the following conclusions can be drawn:

(1) Among the models with extra members arranged, the frames arranged with horizontal members can improve the handling performance at most. The ones with longitudinal members can only improve the handling performance a little, and how the ones with inclined members can improve the handling performance depend on the kart is cornering right of left.

(2) Increasing the kingpin width can more efficiently enhance the torsional stiffness than arranging extra members, but the weight of the frame will be increased. Furthermore, the maximum kingpin width of the kart frame is limited to $1400 \mathrm{~mm}$ according to the CIK-FIA technical regulations [1].

The study on improvement of the torsional stiffness of the go-kart frame with two design concepts has been analyzed systematically in this paper. Since gokarting is a very popular recreation, and usually the kart frame will harden after 
few races, the working life or fatigue strength of the go-kart frame is also an important subject. In addition, the driver's safety and the collision strength of the go-kart are also important factors in this sport, so the crashworthiness of the go-kart with adequate dummy involved is also a topic worth studying. Furthermore, the kart impacting with bumpers attached to the frame, and how to design a bumper to effectively absorb the energy during impact are also an interesting subject in the future.

\section{Acknowledgements}

The authors would like to express the greatest gratitude to the National Science Council of the Republic of China for financially supporting this research under contract No. NSC 93-2745-E-212 -001 -URD, and No. NSC 93-2745-E-212001-URD, and to Da-Yeh University, Taiwan, for extra financially supporting this research under contract No. A-DME-9349.

\section{References}

[1] 2005 CIK-FIA Technical Regulations

[2] L. Solazzi, S. Matteazzi, "Analisi e sviluppi strutturali di un telaio per kart da competizione", AIAS 2002, September 2002.

[3] M. E. Biancolini, R. Baudille, C. Brutti, L. Reccia, "Integrated multibody/FEM analysis of Vehicle Dynamic Behaviour", FISITA Congress, Giugno 2002. 